\title{
LOCAL CONTRACTIONS IN METRIC SPACES ${ }^{1}$
}

\author{
THAKYIN HU AND W. A. KIRK
}

\begin{abstract}
It is shown that a theorem of E. Rakotch for locally contractive mappings can be deduced from Banach's contraction mapping theorem, and a counterexample to an assertion of R. D. Holmes concerning local radial contractions is given.
\end{abstract}

Let $(X, d)$ be a metric space, $k \in(0,1)$, and $g$ a mapping of $X$ into $X$. If for each $x \in X$ there exists a neighborhood $N(x)$ of $x$ such that for each $u, v \in N(x), d(g(u), g(v)) \leqslant k d(u, v)$, then $g$ is said to be locally contractive (with constant $k$ ); if it is assumed only that $d(g(u), g(x)) \leqslant k d(u, x)$ for all $u \in N(x)$, then $g$ is said to be a local radial contraction. These are two of the standard localizations of the contraction mapping condition (cf. Rakotch [3] and Holmes [2], respectively).

It is proved in [3] that if $X$ is a complete metric space and $g: X \rightarrow X$ locally contractive, and if in addition it is the case that for some $x_{0} \in X$ the points $x_{0}$ and $g\left(x_{0}\right)$ are joined by a rectifiable path (i.e., a path of finite length) then $g$ has a unique fixed point in $X$. An example, also given in [3], shows that this pathwise connectedness assumption cannot be dropped. In a related subsequent development, R. D. Holmes has proved [2, Theorem 1] that if $(X, d)$ is connected and locally connected, and if $g: X \rightarrow X$ is a homeomorphism of $X$ onto $X$ which is also a local radial contraction, then there exists a metric $\delta$ on $X$ which is topologically equivalent to $d$ such that $g$ is a contraction globally on $(X, \delta)$. In addition, it is asserted in [2, Corollary, p. 87] that completeness of $(X, \delta)$ follows from completeness of $(X, d)$, and hence that such a mapping $g$ will always have a fixed point via application of the Banach contraction mapping theorem if $(X, d)$ is complete. We give an example below, however, of a complete metric space $X$ and a mapping $g$ such that $g$ and $X$ satisfy all the assumptions of Holmes' Theorem 1, yet $g$ has no fixed point. This shows the assertion to be false. On the other hand, we show that the Rakotch result is a direct consequence of Banach's theorem, even for the more general local radial contractions.

THEOREM 1. Let $(X, d)$ be a complete metric space and $g:(X, d) \rightarrow(X, d) a$ local radial contraction. Suppose for some $x_{0} \in X$ the points $x_{0}$ and $g\left(x_{0}\right)$ are joined by a path of finite length. Then $g$ has a unique fixed point in $X$.

Received by the editors June 17, 1977.

AMS (MOS) subject classifications (1970). Primary 54H25.

Key words and phrases. Local contraction, fixed point theorem, contraction mapping principle.

${ }^{1}$ Research supported in part by National Science Foundation grant MCS 76-03945. 
Our proof of Theorem 1 will use the following fact.

Proposition 1 (Holmes [2]). Let $(X, d)$ be a compact metric space and suppose $g:(X, d) \rightarrow(X, d)$ is a local radial contraction. Then there exist numbers $k \in(0,1), \beta>0$, such that $d(g(x), g(y)) \leqslant k d(x, y)$ for all $x, y \in$ $X$ such that $d(x, y) \leqslant \beta$.

Proof of Theorem 1. Let $\tilde{X}$ be the subset of $X$ consisting of those points which can be joined to $x_{0}$ by a path of finite length. If $x, y \in \tilde{X}$ and if $\alpha$ is a path joining $x$ and $y$, let $l(\alpha)$ denote the length of $\alpha$ and introduce the intrinsic metric $l$ on $\tilde{X}$ by defining

$$
l(x, y)=\inf _{\alpha} l(\alpha)
$$

where $\alpha$ varies over all paths joining $x$ and $y$. We complete the proof by showing:

(1) $g: \tilde{X} \rightarrow \tilde{X}$

(2) $g$ is a contraction mapping on $\tilde{X}$ relative to the metric $l$;

(3) $(\tilde{X}, l)$ is complete.

To prove (1), let $y \in \tilde{X}$ and suppose $\alpha$ is a rectifiable path joining $x_{0}$ and $y$. By Proposition 1 the mapping $g$ restricted to $\alpha$ is locally lipschitzian for some constant $k \in(0,1)$ and a standard argument shows that $g$ maps $\alpha$ onto a path $\beta$ joining $g\left(x_{0}\right)$ and $g(y)$ with $l(\beta) \leqslant k l(\alpha)$. By assumption there exists a rectifiable path $\gamma$ joining $x_{0}$ and $g\left(x_{0}\right)$; thus a rectifiable path joining $x_{0}$ and $g(y)$ is obtained from $\beta \cup \gamma$, proving $g(y) \in \tilde{X}$.

The assertion (2) is also a routine consequence of the locally lipschitzian character of the mapping $g$.

Now suppose $\left\{x_{n}\right\}$ is a Cauchy sequence in $(\tilde{X}, l)$. Since $l(x, y) \geqslant d(x, y)$, $x, y \in \tilde{X}$, it follows that $\left\{x_{n}\right\}$ is also a Cauchy sequence in $(\tilde{X}, d)$; hence there exists $x \in X$ such that $\lim _{n \rightarrow \infty} d\left(x_{n}, x\right)=0$. We shall complete the proof showing that $x \in \tilde{X}$ and $\lim _{n \rightarrow \infty} l\left(x_{n}, x\right)=0$.

Let $\left\{\varepsilon_{i}\right\}$ be a sequence of positive numbers such that $\sum_{i=1}^{\infty} \varepsilon_{i}<\infty$. Since $\left\{x_{n}\right\}$ is Cauchy in $(\tilde{X}, l)$ there exists a strictly increasing sequence $\left\{N_{i}\right\}$ of positive integers such that if $m, n \geqslant N_{i}, l\left(x_{m}, x_{n}\right)<\varepsilon_{i}$. Thus it is possible to obtain a subsequence $\left\{\bar{x}_{n}\right\}$ of $\left\{x_{n}\right\}$ (choosing $\left.n>N_{n}\right)$ such that $l\left(\bar{x}_{n}, \bar{x}_{n+1}\right)<$ $\varepsilon_{n}, n=1,2, \ldots$ For each $n$ there exists a path $\alpha_{n}:[1 /(n+1), 1 / n] \rightarrow(\tilde{X}, d)$ joining $\bar{x}_{n}$ and $\bar{x}_{n+1}$ with length less than $\varepsilon_{n}$. Define the path $\alpha:[0,1] \rightarrow(\tilde{X}, d)$ by taking $\alpha(t)=\alpha_{n}(t)$ if $t \in[1 /(n+1), 1 / n]$ and $\alpha(0)=x$. Clearly $\alpha$ is continuous on $(0,1]$; to see that $\alpha$ is continuous at 0 , let $t_{i} \downarrow 0$ and note that for given $N$, and for $i$ sufficiently large, $t_{i} \in[1 /(n+1), 1 / n]$ for some $n \geqslant N$, from which $d\left(\alpha\left(t_{i}\right), x\right) \leqslant d\left(\bar{x}_{n}, x\right)+\varepsilon_{n}$. From this it follows that $\lim _{i \rightarrow \infty} d\left(\alpha\left(t_{i}\right), x\right)=0$. Moreover, it is clear that $l(\alpha)$ does not exceed $\sum_{i=1}^{\infty} l\left(\bar{x}_{i}, \bar{x}_{i+1}\right)$, and in fact,

$$
l\left(\bar{x}_{n}, x\right) \leqslant \sum_{i=n}^{\infty} l\left(\bar{x}_{i}, \bar{x}_{i+1}\right)=\sum_{i=n}^{\infty} \varepsilon_{i} .
$$

Therefore, $\lim _{n \rightarrow \infty} l\left(\bar{x}_{n}, x\right)=0$. Since $\left\{\bar{x}_{n}\right\}$ is a subsequence of the Cauchy 
sequence $\left\{x_{n}\right\}$ in $(\tilde{X}, l)$, it follows that $\lim _{n \rightarrow \infty} l\left(x_{n}, x\right)=0$.

Although it is possible to give a simple direct proof of Theorem 1 along the lines of Rakotch [3], it has been our primary intention here to illustrate the connection between Theorem 1 and Banach's theorem by clarifying underlying facts. We also remark that implicit in the proof of (3) above is the following fact which may not be generally known (although it is known to be true under an additional assumption (cf. [4, p. 120])). (In this connection, recall that compactness of $(X, d)$ does not imply compactness of $(X, l)$.)

COROllary. Suppose $(X, d)$ is a complete metric space for which each two points are joined by a rectifiable path. Then the space $(X, l)$ is also complete, where $l$ is the intrinsic metric on $X$ induced by $d$.

The EXAmple. We now give an example which shows that the conclusion of Theorem 1 may fail if $x_{0}$ and $g\left(x_{0}\right)$ are merely assumed joined by an arbitrary path rather than a rectifiable path. In addition, our example provides a counterexample to the corollary of $[2$, p. 87$]$.

We re-metrize the real line $R^{1}$ as follows: Let $\left\{\beta_{n}\right\}_{n=-\infty}^{\infty}$ be a strictly increasing doubly infinite sequence of numbers in $(0,1)$. For $x, y \in R^{1}$, define

$$
d(x, y)=\left\{\begin{array}{l}
|x-y|^{\beta_{n}} \quad \text { if } x, y \in[n, n+1] \\
|x-(n+1)|^{\beta_{n}}+(p-1)+|(n+p)-y|^{\beta_{n+p}} \\
\quad \text { if } x \in[n, n+1], y \in[n+p, n+p-1], p>0
\end{array}\right.
$$

It is a straightforward matter to verify that $d$ is a metric (see, for example, the discussion of metric transforms in Blumenthal [1, p. 130]), and clearly $\left(R^{1}, d\right)$ is pathwise connected and complete. Define the homeomorphism $g: R^{1} \rightarrow R^{1}$ by taking $g(x)=x+1$. Then $g$ is locally contractive relative to $d$ for any $k \in(0,1)$. To see this, suppose $x, y \in(n, n+1)$. Then

$$
d(g(x), g(y))=|x-y|^{\beta_{n+1}} \leqslant k|x-y|^{\beta_{n}}=k d(x, y)
$$

provided $|x-y|^{\beta_{n+1}-\beta_{n}} \leqslant k$. Since $\beta_{n+1}-\beta_{n}>0$, such a choice is always possible; indeed, if $d(x, y)<k^{\beta_{n} /\left(\beta_{n+1}-\beta_{n}\right)}$ then $d(g(x), g(y)) \leqslant k d(x, y)$. To deal with the case $x=n$, merely take a $d$-neighborhood of $x$ with radius less than $\min \left\{k^{\beta_{n} /\left(\beta_{n+1}-\beta_{n}\right)}, k^{\beta_{n} /\left(\beta_{n}-\beta_{n-1}\right)}\right\}$.

We note that in the space $\left(R^{1}, d\right)$, all arcs have infinite length.

\section{REFERENCES}

1. L. M. Blumenthal, Theory and applications of distance geometry, Clarendon Press, Oxford, 1953.

2. R. D. Holmes, Fixed points for local radial contractions, Proc. Seminar on Fixed Poini Theory and Its Applications (Dalhousie Univ., 1975), Academic Press, New York, 1976, pp. 79-89. 
3. E. Rakotch, A note on $\alpha$-locally contractive mappings, Bull. Res. Council Israel 40 (1962), 188-191.

4. W. Rinow, Die innere Geometrie der metrischen Räume, Springer-Verlag, Berlin, 1961.

Deparmient of Mathematics, Thi University of Iowa, Iowa Crty, Iowa 52242 (Current address of W. A. Kirk).

Current address (Thakyin Hu): Tam-Kang College of Arts and Sciences, Taipei, Taiwan 